ARTICLE

DOI: $10.1038 / s 41467-018-06872-0$

\title{
Design and self-assembly of hexahedral coordination cages for cascade reactions
}

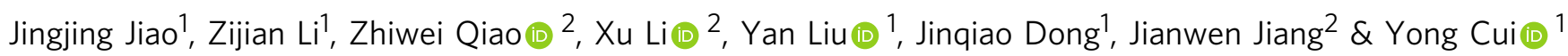

The search for supramolecular reactors that contain no catalytically active sites but can promote chemical transformations has received significant attention, but it remains a synthetic challenge. Here we demonstrate a strategy of incorporating bulky and electro-rich aromatic linkers into metallocages to induce cascade reactions. Two hexahedral cages with a framework formula $\left[\left(\mathrm{Zn}_{8} \mathbf{L}_{6}\right)(\mathrm{OTf})_{16}\right]$ are assembled from six tetrakis-bidentate ligands derived from tetraphenylethylene and eight zinc(II)tris(pyridylimine) centers. The cage cavities can accommodate different molecules such as anthranilamide and aromatic aldehyde through supramolecular interactions, allowing for a cascade condensation and cyclization to produce nonplanar 2,3-dihyroquinazolinones. The reaction is highly efficient with high rate enhancements (up to $k_{\text {cat }} / k_{\text {uncat }}=38,000$ ) and multiple turnovers compared to the bulk reaction mixture. Control experiments and molecular simulations suggest that the acceleration is attributed to inherent strength of binding affinity for reactants and the release of products to establish catalytic turnover is due to the host-guest geometry discrepancy.

\footnotetext{
${ }^{1}$ School of Chemistry and Chemical Engineering and State Key Laboratory of Metal Matrix Composites, Shanghai Jiao Tong University, 200240 Shanghai, China. ${ }^{2}$ Department of Chemical and Biomolecular Engineering, National University of Singapore, Singapore 117576, Singapore. Correspondence and requests for materials should be addressed to Y.L. (email: liuy@sjtu.edu.cn) or to J.J. (email: chejj@nus.edu.sg) or to Y.C. (email: yongcui@sjtu.edu.cn)
} 
S elf-assembled supramolecular containers have attracted growing attention as enzyme mimetics for potential applications in molecular recognition and storage ${ }^{1}$, sensing ${ }^{2}$, catalysis $^{3}$, and drug transporters ${ }^{4}$. Of particular interest is the possibility of performing chemical transformations in confined spaces, in which the relativities and selectivities may be quite different from those in solution. In light of their well-defined yet tunable structures ${ }^{5,6}$, coordination cages provide an ideal platform for designing supramolecular catalysts for chemical transformations ${ }^{3}$. In this respect, pioneering works have been demonstrated by Fujita ${ }^{7,8}$ and Raymond ${ }^{9,10}$. Some representative reactions include Diels-Alder ${ }^{11,12}$, epoxidation ${ }^{13}$, the aza-Cope rearrangement ${ }^{14}$, Knoevenagel reaction ${ }^{15}$, Nazarov cyclization ${ }^{16}$, sigmatropic rearrangements ${ }^{17}$, and a few others ${ }^{18-23}$. Despite that some examples are known for efficient bimolecular reactions in containers without catalytically active sites, there is a great need for the design of new supramolecular capsules to promote more complicated reactions $s^{7,8,24,25}$. Moreover, the range of reaction types is narrow, limiting their use in practical organic synthesis. Cascade or sequential catalytic reactions, as sophisticatedly manipulated by nature, are of great value because such processes can guide the reactive intermediates to the targeted products via consecutive reactions ${ }^{26,27}$. However, it remains unexplored to rationally design catalytic cages for tandem reactions ${ }^{28,29}$. In artificial systems, product inhibition poses another challenge in establishing a catalytic cycle ${ }^{3}$. To address these issues, here we report a strategy of incorporating bulky and electro-rich aromatic linkers into metallocages to induce twocomponent cascade reactions and reduce product inhibition.

Coordination cages constructed from organic ligands with extended aromatic panels can provide favorable interactions with aromatic molecules due to high $\pi$-electron density of assembled walls $^{30,31}$. We envisioned that specific aromatic-aromatic and/or edge-to-face aromatic interactions may be used for hosts to concentrate aromatic reactants, regulate their orientations and even promote catalytic reactions to generate products that have weak host-guest interactions and can be expelled to allow catalytic turnover ${ }^{3}$. Tetraphenylethylene (TPE) and its derivatives have recently attracted much attention owing to their rich electrochemical and excited state properties ${ }^{31}$ and particularly have been employed for designing cages and metallacycles with aggregation-induced emission ${ }^{32,33}$. Considering their $\pi$-electronrich and structural flexibility, in this study we select two TPEderived tetraamines as ligands for subcomponent self-assembly of octanuclear $\mathrm{Zn}_{8} \mathrm{~L}_{6}$ cages with tunable cavity sizes (Fig. 1). We demonstrate the TPE-based cages could control uptake and release of guests with different shapes and accelerate catalytically the cascade condensation and cyclization of anthranilamide and aromatic aldehydes to nonplanar 2,3-dihyroquinazolinones, with high rate enhancements in comparison to the bulk reaction mixture. The present two cages are rare examples of hollow hosts that can efficiently discern between substrate and product, allowing weak product binding and efficient catalysis.

\section{Results}

Synthesis and characterization. The ligand $\mathbf{L}_{1}$ was synthesized according to the reported procedure ${ }^{34}$, and $\mathbf{L}_{2}$ was synthesized by the Suzuki cross-coupling reaction of (4-nitrophenyl)boronic acid pinacol ester and tetra(4-bromophenyl)ethylene and followed by reduction with $\mathrm{Pd} / \mathrm{C}$ (Supplementary Figs. 10-12). As shown in Fig. 1, the cages TPE-1 and TPE-2 with the general formula $\left[\left(\mathrm{Zn}_{8} \mathbf{L}_{6}\right)(\mathrm{OTf})_{16}\right] \cdot \mathrm{G} \quad(\mathrm{G}=$ guest molecule $)$ were obtained by heating $\mathrm{Zn}(\mathrm{OTf})_{2}, \mathbf{L}_{1}$ or $\mathbf{L}_{2}$ and 2-formylpyridine in $\mathrm{CH}_{2} \mathrm{Cl}_{2}$ and $\mathrm{CH}_{3} \mathrm{CN}$ at $70{ }^{\circ} \mathrm{C}$ with 64 and $35 \%$ yield, respectively. Single crystals of the cages were obtained by diffusion of a mixed a
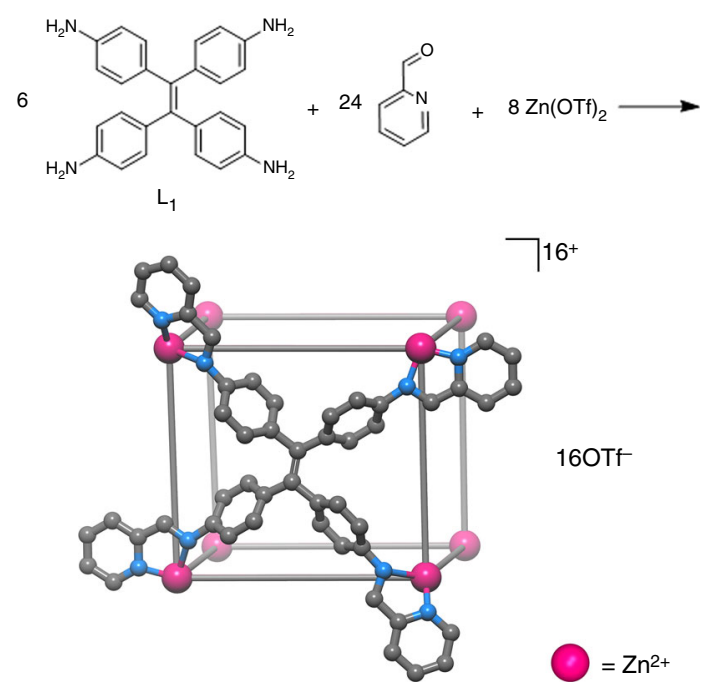

b
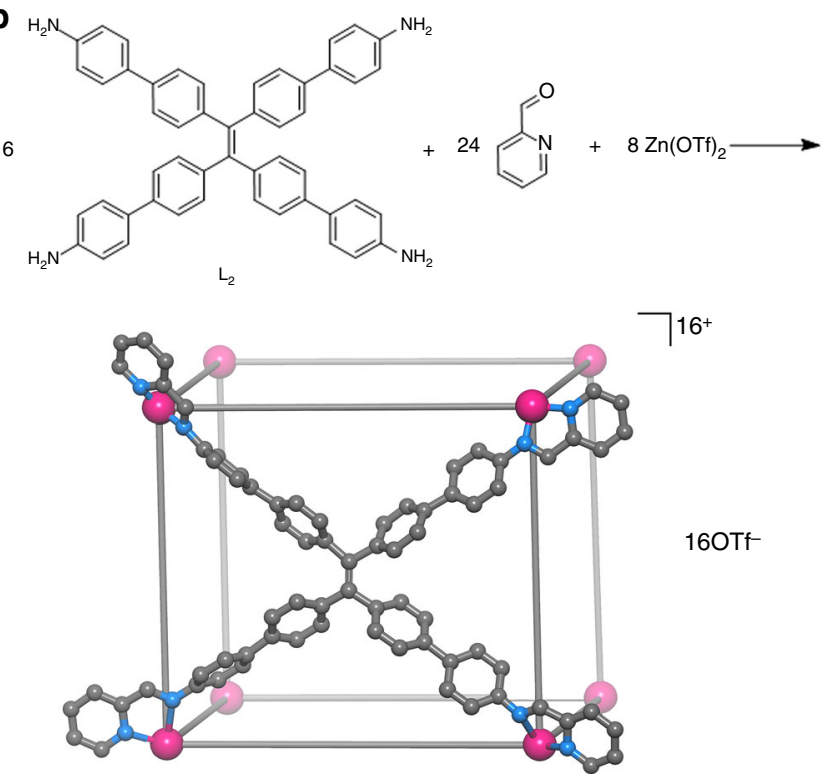

$16^{+}$

$16 \mathrm{OTf}$

Fig. 1 Subcomponent self-assembly. a TPE-1 and b TPE-2

solvents of $\mathrm{Et}_{2} \mathrm{O}$ and THF or 1,4-dioxane and THF (1/1, v/v) into a dilute and saturated $\mathrm{CH}_{3} \mathrm{CN}$ solution. The formulations were supported by the results of microanalysis, IR spectroscopy, ${ }^{1} \mathrm{H}$ and ${ }^{13} \mathrm{C}$ NMR, ${ }^{1} \mathrm{H}$ COSY, NOESY, Quadrupole time-of-flight mass spectrometry (Q-TOF-MS), thermogravimetric analyses (TGA) and single-crystal X-ray diffraction.

The ${ }^{1} \mathrm{H}$ and ${ }^{13} \mathrm{C}$ NMR spectra of each cage displayed only one set of ligand resonances in solution, suggesting the formation of a discrete and highly symmetric assembly (Supplementary Figs. 13 and 14); most of these signals have a slight downfield shift with respect to the free ligand. The $C_{2}$-symmetry of the TPE ligand is preserved in the cage, as could be deduced from the number of signals in the NMR spectra. A single peak was observed for each proton of the ligand, with ${ }^{1} \mathrm{H}-{ }^{1} \mathrm{H}$ COSY, NOESY data allowing assignment of each signal (Supplementary Figs. 18 and 19). In diffusion-ordered NMR spectroscopy ( ${ }^{1} \mathrm{H}$-DOSY) for TPE-1 (Supplementary Fig. 20a), the observation of a distinct band at log $D=-9.51\left(\mathrm{~m}^{2} \mathrm{~s}^{-1}\right)$ with a hydrodynamic radius of about $11.76 \AA$ indicated the formation of single product. Similarly, ${ }^{1} \mathrm{H}$-DOSY of TPE-2 showed one band at $\log D=-9.61\left(\mathrm{~m}^{2} \mathrm{~s}^{-1}\right)$ with a hydrodynamic radius of about $14.79 \AA$ indicating a slightly larger size than TPE-1 (Supplementary Fig. 20b). Q-TOF-MS analysis 
provided further support for the existence of multitetraphenylethene assemblies, and a clean spectrum was obtained with peaks displaying the expected isotopic patterns at 1083.96, 1331.55, and 1700.41 for TPE-1 belonging to $\left[\mathrm{Zn}_{8}\left(\mathrm{~L}_{1}\right)_{6} \cdot x \mathrm{CH}_{3} \mathrm{CN} \cdot y \mathrm{THF} \cdot z \mathrm{H}_{2} \mathrm{O} \cdot(16-n) \mathrm{OTf}\right]^{n+}(n=4-6$, Supplementary Fig. 21) and 650.41, 795.89, and 916.64 for TPE-2 belonging to $\left[\mathrm{Zn}_{8}\left(\mathbf{L}_{2}\right)_{6} \cdot x \text { dioxane } \cdot y \mathrm{THF} \cdot z \mathrm{CH}_{3} \mathrm{CN} \cdot(16-n) \mathrm{OTf}\right]^{n+}$ $(n=9,10,12$; Supplementary Fig. 22). TGA revealed that guest molecules in crystalline TPE- 1 and TPE- 2 could be removed in the temperature range from 60 to $160^{\circ} \mathrm{C}$ and the materials started to decompose at $\sim 460^{\circ} \mathrm{C}$ (Supplementary Fig. 26).

X-ray crystallography. Single-crystal X-ray diffraction study on TPE-1 and TPE-2 revealed the formation of porous hexahedral cages. TPE-1 crystallizes in the chiral hexagonal space group $\mathrm{Pb}_{2} 22$, with one-fourth of the formula unit in the asymmetric unit and three crystallographic $C_{2}$-axes passing through three opposite pairs of the ligands. Each of the eight tris(pyridylimine)zinc(II) vertices has an octahedral coordination geometry, with the $\mathrm{Zn}$ center chelated by three ligands. The $\mathrm{Zn}$ ions in one cage have the same $\Delta$ or $\Lambda$ configuration. The $\mathrm{Zn}-\mathrm{N}$ bond lengths range from 2.09 (2) to 2.27(2) $\AA$, which are in good agreement with those reported for related $\mathrm{Zn}$-pyridylimine complexes.

Each of the six TPE ligands lying on a two-fold axis coordinates to four zinc atoms through its four chelating pyridylimine groups. The four benzene rings in TPE are non-coplanar, with dihedral angles ranging from 62.9 to $87.6^{\circ}$, giving rise to the four-bladed propeller structure in the cage. This arrangement of metal ions and coordination ligands thus leads to a molecular cube with the eight corners occupied by the zinc ions and the six faces by six TPE ligands. The $\mathrm{Zn}-\mathrm{Zn}$ separations along the cage edges are from $10.27(3)$ to $11.47(3) \AA$ (Supplementary Fig. 1a) and the ethylene bond separations for TPE between opposite faces are from $10.81(5)$ to $11.75(11) \AA$. The cavity of the cage has inner voids around $522.3 \AA^{3}$ (calculated by assuming that the windows are blocked and considering the van der Waals radius) (Fig. 2a, c; Supplementary Fig. 2). The benzene groups of $\mathbf{L}_{1}$ slightly protrude into the cavity with an irregular window $(3.7 \times 7.8$ $\AA^{2}$ ), generating portals to allow the ingress/egress of guests (Supplementary Fig. 1b).

The large cage TPE- 2 crystallizes in space group $P \overline{1}$, with the whole formula unit in the asymmetric unit. Due to the increased rotational freedom from $\mathbf{L}_{1}$ in TPE-1 to $\mathbf{L}_{2}$ in TPE-2, the $C_{2}$ symmetry was broken. TPE-2 has a similar hexahedral structure assembled from eight zinc ions and six $\mathbf{L}_{2}$ ligands. The $\mathrm{Zn}-\mathrm{Zn}$ distances along the edges of the hexahedron range from 14.59(5) to $18.34(7) \AA$ (Supplementary Fig. 3a), and the ethylene bond separations between the opposite faces are in the range of 17.10 (3) $-18.45(3) \AA$ A. Space-filling representations of TPE-2 clearly show the formation of a large porous cage with a volume of about 2222.4 $\AA^{3}$ (Fig. 2b, d; Supplementary Fig. 4), and the longer ligands $\mathbf{L}_{2}$ reduce an irregular wide window $\left(13.7 \AA \times 6.4 \AA^{2}\right)$ (Supplementary Fig. 3b). So, TPE-2 offers a much larger and flexible cavity than TPE-1. Notably, in both cases, the $\mathrm{C}-\mathrm{H}$ bonds in the highly twisted aromatic rings are oriented toward the inside of cavities, offering potential for strong supramolecular interactions and the encapsulation of guest molecules. This is different from most of the rigid coordination cages for host - guest chemistry ${ }^{7,9,21}$.

Supramolecular catalysis. The present porous hexahedral cages featuring rich $\pi$-electron density may accommodate aromatic guests, which is beneficial for chemical transformation and catalysis in the cavities. We therefore employed the two cages for supramolecular catalysis by taking advantage of their hydrophobic and flexible cavities with tunable size. In this study, we focus on the catalytic synthesis of 2,3-dihydroquinazolinones, which are an important class of fused heterocycles due to their pharmacological activities, such as antitumor, analgesic, antifibrillatory, antibiotic, antispermatogenic, and vasodilatory effi$\mathrm{cacy}^{35}$. The catalytic activities of the cages were evaluated by using anthranilamide (3a) and 4-fluorobenzaldehyde (4b) as substrates. After screening various reaction conditions including catalyst loading, reaction temperature, and solvent (Supplementary Table 8 ), we found that, in the presence of $0.1 \mathrm{~mol} \%$ of TPE1, the reaction of anthranilamide and 4-fluorobenzaldehyde proceeded smoothly in a mixture of $\mathrm{CH}_{3} \mathrm{CN}$ and toluene at $40^{\circ} \mathrm{C}$. Notably, a lower catalyst loading $(0.05 \mathrm{~mol} \%)$ can be used, but prolonged reaction time $(45 \mathrm{~h})$ was required. We measured the reaction rate under this condition by ${ }^{1} \mathrm{H}$ NMR spectroscopy: the starting material transformed into intermediate $\mathbf{5 b}$ and product 6b nearly completely in $3 \mathrm{~h}$ and completed within $11 \mathrm{~h}$ to furnish the targeted 2,3-dihydroquinazolinone (6b) in 95\% yield. Besides, the reactions of anthranilamide with other aromatic aldehydes such as benzaldehyde, 4-methylbenzaldehyde, and 4methoxybenz aldehyde can also be catalyzed by TPE-1, which afforded $76-81 \%$ yields of the products (Table 1 ). Anthranilamide with electron-donating or -withdrawing substituents (-Me or $-\mathrm{Cl}$ ) on the phenyl group afford the targeted products with excellent yields (entries 19 and 21, Table 1).

Under identical conditions, TPE-2 is found to be a more efficient catalyst than TPE-1 for the above sequential reactions (Fig. 3); probably due to that it has a much larger pore for substrate exchange. Specifically, the reactions catalyzed by $0.1 \mathrm{~mol}$ \% loading of TPE-2 were complete within $7 \mathrm{~h}$ monitored by ${ }^{1} \mathrm{H}$ NMR spectroscopy (Fig. 3b, c), affording $87-99 \%$ isolated yields. A ${ }^{1} \mathrm{H}$ NMR study of the precipitate, which was recovered from the reaction mixture by adding ethyl ether, did not show any trace of the encapsulated product, signifying the weak interaction of the product with the cage cavity. It was apparent that sequential reactions of anthranilamide and aldehyde were accelerated by the cages. To measure the rate constant in the presence of cage $\left(k_{\text {cat }}\right)$ with little interference from the background reaction $\left(k_{\text {uncat }}\right)$, we assessed the model of Michaelis-Menten scheme (Supplementary Figs. 29 and 30 $)^{21}$. As expected, kinetics achieved saturation at high substrate concentrations and showed a first dependence on the catalyst loading (Supplementary Fig. 31 ). The $k_{\text {cat }} / k_{\text {uncat }}$ ratios were found to be $1.1 \times 10^{4}$ and $3.8 \times 10^{4}$ for TPE-1 and TPE-2, respectively. Therefore, the cages reported here can be potentially acted as functional enzyme mimic in catalysis ${ }^{21,36}$. The catalytic activities observed for the two cages are comparable well with those reported for strong Lewis acids such as $\mathrm{Zn}(\mathrm{OTf})_{2}$, Sc $(\mathrm{OTf})_{3}{ }^{37}$ and $\mathrm{Ga}(\mathrm{OTf})_{3}{ }^{38}$ or strong Brønsted acids such as sulfonic acid $^{35}$ and phosphoric acid ${ }^{39}$ at $1.0 \mathrm{~mol} \%$ catalyst loading (Supplementary Fig. 33). When the loading reduce to $0.1 \mathrm{~mol} \%$, all of these catalytic systems failed to promote the cyclization and imine was observed as a major product. The rate accelerations of the catalyzed reaction over the uncatalyzed reaction are on the order of $10^{4}$, which are well comparable with those reported for supramolecular catalysis in metal-ligand hosts (Supplementary Table 5) $)^{9,12}$

To prove that the efficient catalysis occurs predominantly within the cage cavities, we conducted a series of extensive experimental studies. First, we added an excess of a strongly binding competing guest $\left(2.5 \mathrm{mmol}\right.$ pyrene, $K_{\mathrm{a}}=2.8 \times 10^{5} \mathrm{M}^{-1}$ and $2.0 \times 10^{5} \mathrm{M}^{-1}$ for TPE-1 and TPE-2, respectively) (Supplementary Fig. 44). With this inhibitor present, the reaction cannot proceed at all because the competing guest prevented substrate binding in the cage cavity. Second, control experiments showed that, in the absence of the cages, the reaction of $\mathbf{3 a}$ and $\mathbf{4 b}$ only gave the imine intermediate about $47 \%$ yield at $40{ }^{\circ} \mathrm{C}$ after $14 \mathrm{~h}$ 
a

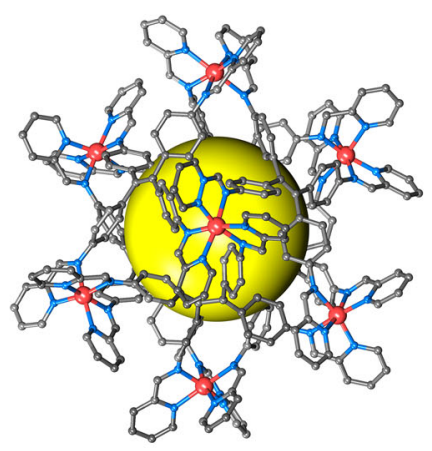

b

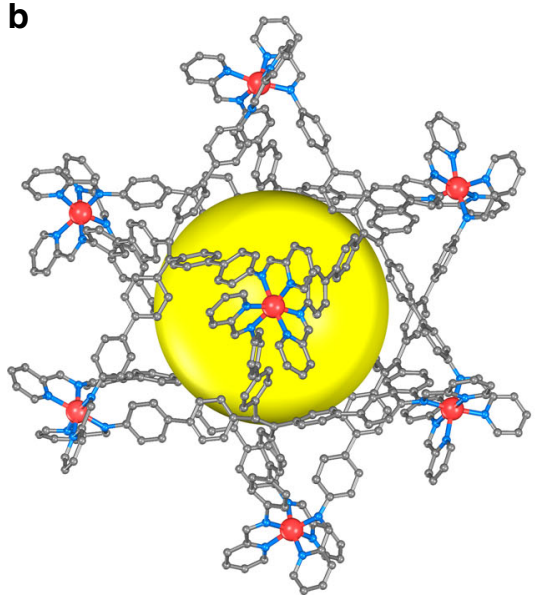

c

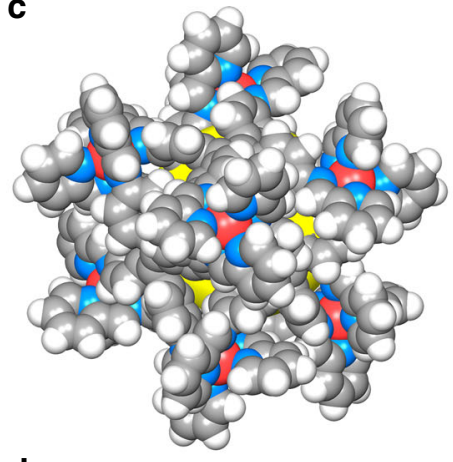

d

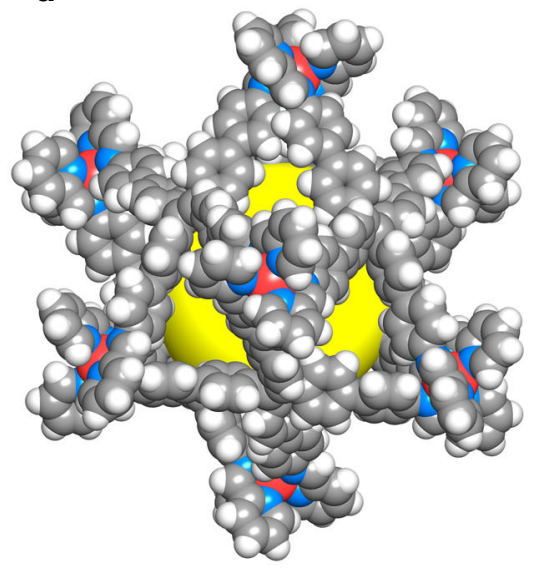

Fig. 2 Single-crystal X-ray structures. a TPE-1, b TPE-2; c, d their space-filling models (the cavities are highlighted by yellow spheres). Color coding: Orange, Zn; Blue, N; Gray, C; White, H

(Table 1, entries 5 and 6; Supplementary Fig. 28). Meanwhile, when 2-formylpyridine $(2.4,240$, and $24,000 \mathrm{~mol} \%), \mathrm{Bu}_{4} \mathrm{NOTf}$ (1.6 $\mathrm{mol} \%$ ), the ligand $\mathbf{L}_{1}\left(\right.$ or $\mathbf{L}_{2}, 0.6 \mathrm{~mol} \%$ ) or a mononuclear tris (pyridylimine)zinc(II) complex $(0.8 \mathrm{~mol} \%)$ (Supplementary Figs. 5, 15 and 23), prepared from 2-formylpyridine and $p$ toluidine was used, no sequential reaction was observed in each case (Table 1, entries 7-10). Furthermore, inductively coupled plasma emission spectrometry (ICP-AES) of the aqueous phase after extracting the reaction mixture indicated 0.01 and $0.02 \%$ loss of Zn ions for TPE-1 and TPE-2, respectively, which excludes the catalytic effect of the free $\mathrm{Zn}$ ions. Third, competitive size selectivity studies were also performed. When 1formylnaphthalene that contains two aromatic rings was employed, TPE-2 efficiently catalyzed the reaction (95\% yield), but TPE-1 cannot promote the reaction at all (Table 1, entries 15 and 16). However, when sterically bulky 9-anthracenealdehyde bearing three aromatic rings was employed (Supplementary Fig. 9), both TPE-1 and TPE-2 cannot promote the reaction, probably due to that the bulky substrate cannot enter into the cavities through the windows (Table 1, entries 17 and 18). Both TPE-1 and TPE-2 have an irregular window with dimensions about $3.7 \times 7.8 \AA^{2}$ and $6.4 \times 13.7 \AA^{2}$, respectively. Despite the well-defined structure, the host ligand framework and the cage cavity are flexible, especially in which the aromatic rings can rotate to adjust portal sizes, and so the substrates such as $\mathbf{3 a}$ $\left(5.8 \times 6.0 \AA^{2}\right)$ and $4 \mathbf{b}\left(6.0 \times 8.5 \AA^{2}\right)$ with sizes smaller and even slightly larger than the portals can enter the cavities from windows ${ }^{40,41}$. However, the substrates are too large, so they cannot enter the cage cavities [1-formylnaphthalene $\left(8.0 \times 8.5 \AA^{2}\right)$ vs. TPE-1 and 9-anthracenealdehyde $\left(8.0 \times 10.9 \AA^{2}\right)$ vs. TPE-1 and TPE-2]. Taking together, the above results suggested that the sequential condensation and cyclization reaction was indeed associated with the substrates being bound in the cage cavity. We demonstrated that the catalytic reaction occurs with a lot of turnovers. To a solution of TPE-1 (0.1 mol\% loading) in $\mathrm{CH}_{3} \mathrm{CN}$ and toluene, we added several successive portions of anthranilamide and 4-fluorobenzaldehyde, waiting until each aliquot had completely reacted before adding the next. We can see from Supplementary Fig. 32 that, after multiple additions of reactants, the reaction profile remained unchanged, and so there is no detectable change in activity after five and even ten turnovers. Moreover, after conversion of all substrates to the product, the cages were recovered and found to retain the structure intact, as evidenced by ${ }^{1} \mathrm{H}$ NMR, TOF mass, and UV-Vis spectra (Supplementary Figs. 16, 17, 24, 25 and 27).

Host-guest interactions. To further understand the host-guest interactions, we studied the ability of the cages to encapsulate reactants and products by ${ }^{1} \mathrm{H}$ and ${ }^{19} \mathrm{~F}$ NMR, IR and UV-Vis titration. First, in the presence of the reactants $\mathbf{3} \mathbf{a}$ and $\mathbf{4 b}$, we observed the downfield shifts of ${ }^{1} \mathrm{H}$ NMR proton signals of reactants (Supplementary Figs. 34 and 35). For example, in a solution of TPE-1 and $\mathbf{3 a}$ or $\mathbf{4 b}$, a 0.17 ppm downfield shift was observed for the proton on the aromatic ring of $3 \mathbf{a}$ at $6.52 \mathrm{ppm}$ and $0.04 \mathrm{ppm}$ downfield shift for the proton on $\mathbf{4 b}$ at $6.36 \mathrm{ppm}$ (Supplementary Fig. 34), and in a solution of TPE-2 and $\mathbf{3 a}$ or $\mathbf{4 b}$, a $0.10 \mathrm{ppm}$ downfield shift was observed for the proton on the aromatic ring of $3 \mathrm{a}$ at $6.69 \mathrm{ppm}$ and $0.03 \mathrm{ppm}$ downfield shift for the proton on $\mathbf{4 b}$ at $7.40 \mathrm{ppm}$ (Supplementary Fig. 35). The downfield shift of ${ }^{19} \mathrm{~F}$ peak of $\mathbf{4 b}$ at $104.61 \mathrm{ppm}$ in the presence of TPE-1/TPE-2 to $78.66 \mathrm{ppm}$ indicates the encapsulation behavior (Supplementary Fig. 36). The host-guest interactions were further confirmed by ${ }^{1} \mathrm{H}$-DOSY in 50:50 (v/v) DMSO- $d_{6} / \mathrm{CD}_{3} \mathrm{CN}$, in which the diffusion coefficients were single set of resonances in the presence of substrates (Supplementary Figs. 37 and 38). For 




the mixture system of $\mathbf{3 a}+\mathbf{4} \mathbf{b}$, both the same value of diffusion coefficient for the substrate mixture and TPE-1/TPE-2 and crossover signals for protons of $\mathbf{3 a} / \mathbf{4} \mathbf{b}$ with protons on the benzene rings of TPE-1/TPE-2 indicated simultaneous encapsulations of the two substrates in the cage (Supplementary Figs. 3739). It should be noted that guest molecules trapped by a host cavity typically exhibit upfield shifts in ${ }^{1} \mathrm{H}$ NMR, although trapped molecules that display upfield shifts were also observed ${ }^{25,42}$. In this work, the cages were constructed with TPE units as faces that contribute a great electron conjugate system around the periphery of cages. When guest molecules moved into the cavity, they may be in the de-shielding distinction of benzene rings. Besides, the formation of favorable $\mathrm{CH} \cdots \pi, \mathrm{CH} \cdots \mathrm{N}$ and $\mathrm{CH} \cdots \mathrm{O}$ interactions upon encapsulation also provided a plausible structural rationale for the downfield shifts.

UV-Vis titration experiments were performed to study the guest binding ability of the cages (Supplementary Figs. 40 and 41). As shown in Fig. 4, a better fit was obtained to a 1:1 host -guest isotherm, and the associate constants $\left(K_{\mathrm{a}}\right)$ were found to be $4.0 \times 10^{4}$ and $1.2 \times 10^{4} \mathrm{M}^{-1}$ for TPE-1 and the analytes $3 \mathbf{a}$ and $4 \mathbf{b}$, respectively, and $1.5 \times 10^{4}$ and $1.0 \times 10^{4} \mathrm{M}^{-1}$ for TPE- 2 and $3 \mathbf{a}$ and $\mathbf{4 b}$, which are much higher than the $K_{\mathrm{a}}$ values of 1191 and $1754 \mathrm{M}^{-1}$ observed for the two cages and the intermediate $\mathbf{5 b}$, and 874 and $215 \mathrm{M}^{-1}$ observed for the two cages and the product 6b (Supplementary Figs. 42 and 43), respectively. The quite different $K_{\mathrm{a}}$ values suggested that the cages catalyzed the reaction a

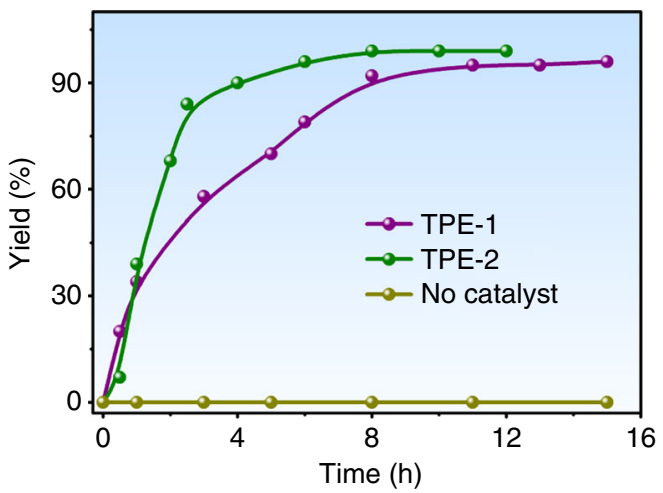

b<smiles>NC(=O)c1ccccc1/N=C/c1ccc(F)cc1</smiles>

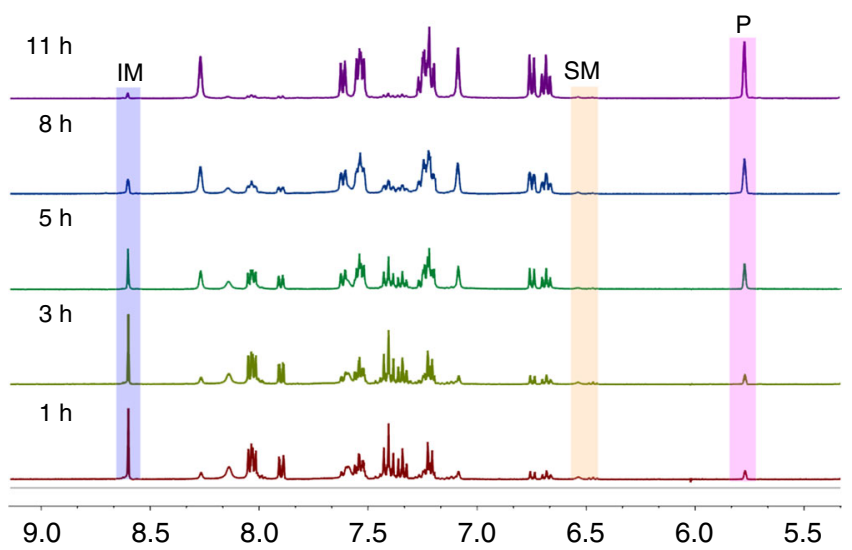

C

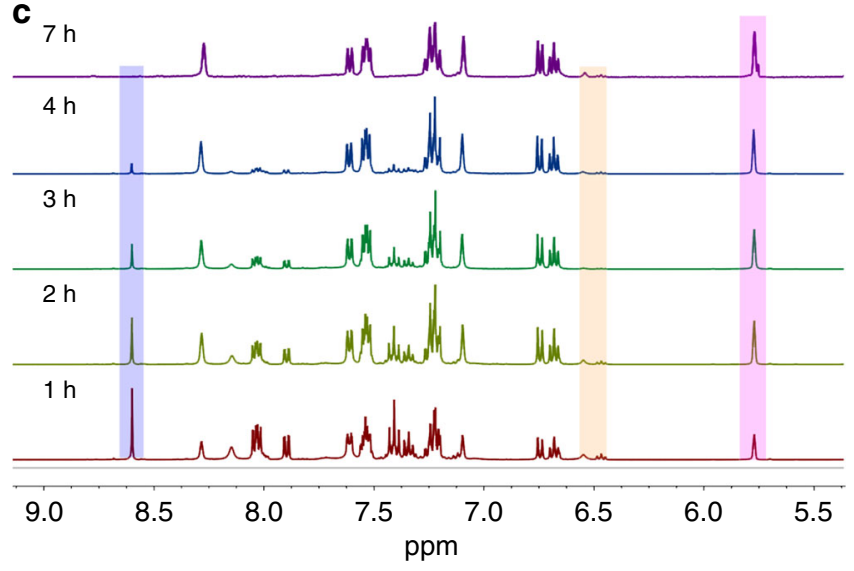

Fig. 3 Kinetic results of the catalysis. a Kinetic curves obtained with $0.1 \mathrm{~mol}$ $\%$ of the cage and without catalyst in the sequential condensation and cyclization of $\mathbf{3} \mathbf{a}$ and $\mathbf{4} \mathbf{b} ; \mathbf{b}, \mathbf{c}$ evolution of ${ }^{1} \mathrm{H}$ NMR spectra during the reaction for TPE-1 and TPE-2 [SM = starting material (3a); IM = intermediate $(\mathbf{5 b}) ; P=$ product $(\mathbf{6 b})]$

as a turnover process based on uptake of the substrate and release of the product. The formation of host-guest adducts was also revealed by solid-state IR spectra, which showed the characteristic peaks of $v(\mathrm{~N}-\mathrm{H})$ at $\sim 3440 \mathrm{~cm}^{-1}$ and $v(\mathrm{C}=\mathrm{O})$ at $\sim 1697 \mathrm{~cm}^{-1}$ for 3a/4b@TPE-1/2 (Supplementary Figs. 45 and 46). While no such characteristic stretching bands due to $\mathbf{6 b}$ were detected 

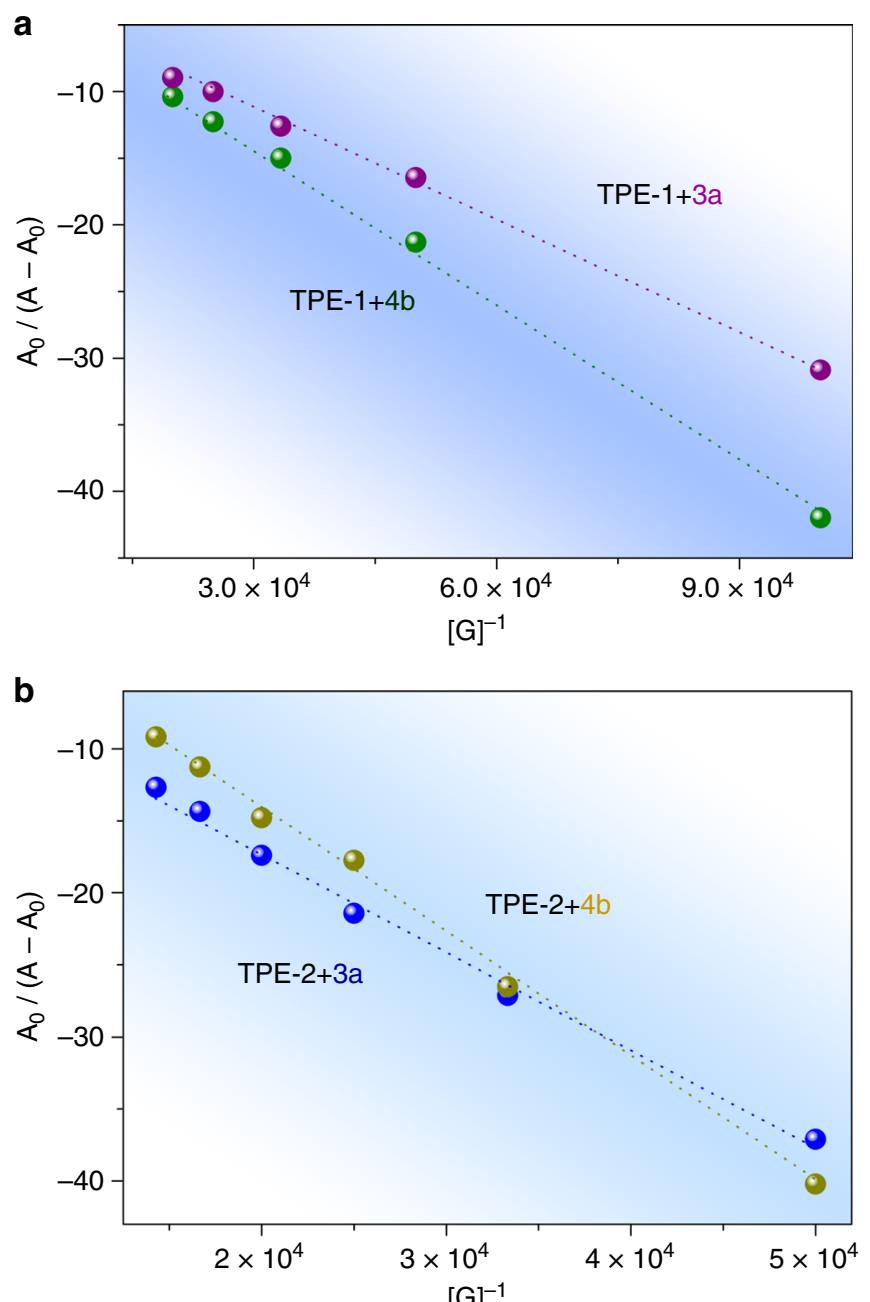

Fig. 4 Benesi-Hildebrand plots. a TPE-1 titration with $\mathbf{3 a}$ and $\mathbf{4 b}$; $\mathbf{b}$ TPE-2 titration with $\mathbf{3} \mathbf{a}$ and $\mathbf{4} \mathbf{b}$. The plots were obtained by using a UV-Vis titration experimental method in $\mathrm{CH}_{3} \mathrm{CN}$ at r.t

(Supplementary Fig. 47), indicating that the product was not adsorbed in the cages.

Molecular dynamic simulation. In order to provide microscopic insight into the cavity-confined effect, molecular dynamics (MD) simulations with the universal force field ${ }^{44}$ were conducted to study the origin of host-guest complexes and estimate their interaction energies under no solvent environment. As shown in Supplementary Fig. 6, both the substrates $\mathbf{3 a}$ and $\mathbf{4 b}$ can be trapped in the cavity of TPE-1 or TPE-2. Each of them is involved with several host-guest interactions including $\mathrm{CH} \cdots \pi$ interactions (2.68-3.32 $\AA$ ) and hydrophobic $\mathrm{CH} \cdots \mathrm{N}$ and $\mathrm{CH}$... O interactions (2.35-3.78 $\AA$ ). Importantly, the cavity-confined microenvironment triggered the inherent strength of binding affinity between the two reactants via $\pi \cdots \pi$ and hydrophobic interactions $(2.65 \sim 3.62 \AA)$. For the substrates $\mathbf{3 a}+\mathbf{4 b}, \mathbf{3} \mathbf{a}+\mathbf{4 e}$ and $\mathbf{3 a}+\mathbf{4 f}$ in TPE-1, the binding energies were calculated to be $-34.98,44.39$, and $52.67 \mathrm{kcal} \mathrm{mol}^{-1}$, respectively (Supplementary Fig. 7 and Supplementary Table 7). The negative value of -34.98 for $\mathbf{3 a}+$ $\mathbf{4 b}$ suggests that the confinement in TPE-1 is favorable for host - guest interaction and subsequent condensation reaction. With increasing molecular size of $\mathbf{4}$, the energies in TPE-1 becomes positive, which implies that the cage is not sufficiently large to

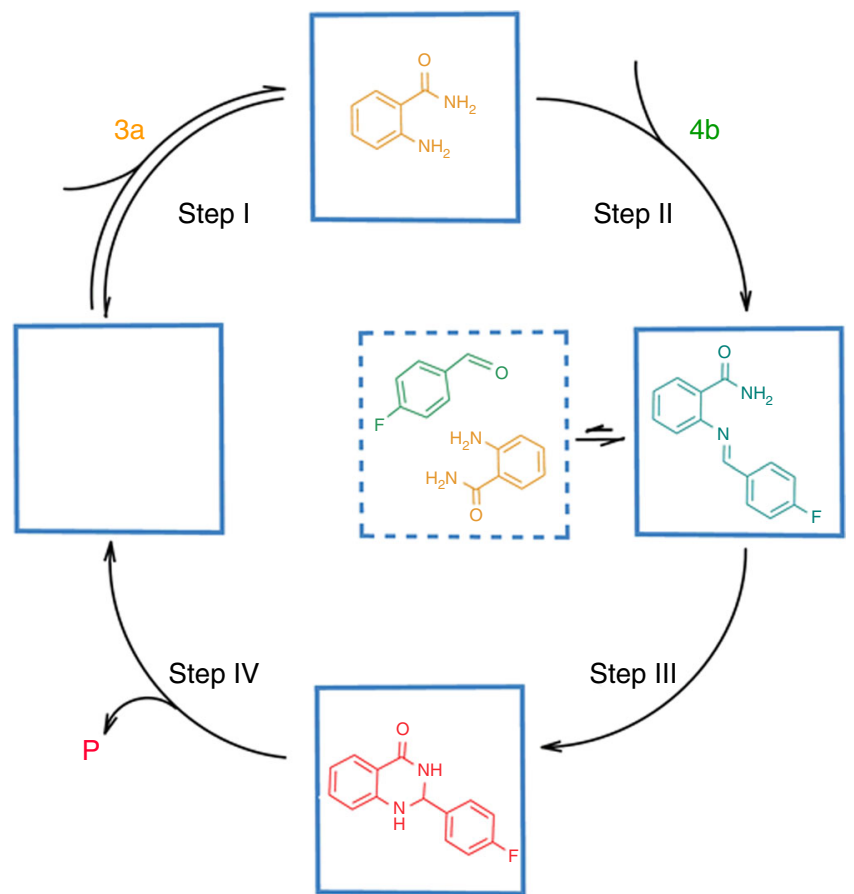

Fig. 5 Representation of the sequential condensation and cyclization of anthranilamide with aldehyde in the cage (the square represents the cage; 3a and $\mathbf{4} \mathbf{b}$ stand for the substrates and $\mathbf{P}$ stands for the product)

accommodate $3 \mathbf{a}+\mathbf{4 e}$ or $\mathbf{3 a}+\mathbf{4 f}$, thereby leading to the steric hindrance inhibition of the reaction. Unlike TPE-1, the energies are found to be $-126.95,-94.65$, and $18.65 \mathrm{kcal} \mathrm{mol}^{-1}$ for $\mathbf{3 a}+$ $\mathbf{4 b}, \mathbf{3 a}+\mathbf{4 e}$ and $\mathbf{3 a}+\mathbf{4 f}$ in TPE-2, respectively. Obviously, TPE-2 has a larger cavity to accommodate $\mathbf{3 a}+\mathbf{4 e}$ with stable interaction and thereby can promote the reaction (in addition to $\mathbf{3 a}+$ 4b). Nevertheless, the bulky reactants $3 \mathbf{a}+\mathbf{4 f}$ cannot fit into the cavity of TPE-2 and their condensation reaction is impeded.

It should be noted that the binding energies from simulations were based on an implicit solvent model. If the solvent molecules were included, the simulations would be quite time-consuming. Therefore, there is a large difference in the binding energies of $\mathbf{3 a}+$ 4b with TPE-1 and TPE-2. In experiments, the solvent was present and, to a large extent, screened the binding, thus leading to a small difference in the associate constants obtained from UV-Vis titration. Nevertheless, these calculated interaction energies are consistent with the experimental observations of cavity-confined effect in the two cages. We believe that rate acceleration of this reaction is associated with the enhanced binding of the reactants in the confined cavity.

Proposed catalytic mechanism. Based on the above experimental studies and molecular simulations, we propose the following reaction mechanism (Fig. 5) and attribute the cage cavities to coencapsulate the two different reactants to promote the reaction. First, according to the UV-Vis titration results, both cages prefer to accommodate anthranilamide $3 \mathbf{a}$ (higher $K_{\mathrm{a}}$ than reactant $\mathbf{4 b}$ ) within their hydrophobic cavities (Step I). Second, the reactant $\mathbf{4 b}$ enter cavity and stack with encapsulated $\mathbf{3 a}$ face to face in the most stable configuration. The model of the host-guest binding system was conducted by molecular dynamic simulations revealing the enough negative binding energy (Supplementary Fig. 6). This special binding event in the cavity may reduce the reaction energy barrier, leading to transformation of the intermediate $\mathbf{5 b}$ immediately (Step II). Third, the reaction of a subsequent intramolecular nucleophilic attack of the amide nitrogen 
on the activated imine group is followed by a 1,5-proton transfer to yield the final product in cages (Step III). The rich electronic and conjugate TPE faces of the cages may facilitate the deprotonating of the amide nitrogen via supramolecular interactions to render it more nucleophilic in the cyclization ${ }^{43}$. This is similar to the same cyclization reaction catalyzed by Lewis acids ${ }^{40}$ or Brønsted acids ${ }^{37}$ (Supplementary Fig. 33). However, further study is greatly needed to understand the tandem reaction mechanism with a coordination cage. Fourth, the sequential reaction thus breaks the planarity of aromatic substrates (Supplementary Fig. 8), promoting dissociation of product to allow catalytic turnover (Step IV). The weak product binding was evidenced by the much smaller associate constants with cages than reactants $\mathbf{3 a}$ and $\mathbf{4 b}$ in UV-Vis titrations (Supplementary Figs. 40 and 41). Besides, we did not observe the reaction rate decreasing with time, which means that the reaction cannot be inhibited by accumulation of product.

\section{Discussion}

We have described the design and preparation of two TPE-faced hexahedral $\mathrm{Zn}_{8} \mathrm{~L}_{6}$ coordination cages with tunable cavity sizes by subcomponent self-assembly. The flexible and hydrophobic cavities of the two cages favor inclusion of aromatic substrates via $\mathrm{CH} \cdots \pi$ and hydrophobic interactions and can efficiently encapsulate anthranilamides and aromatic aldehydes and accelerate catalytically a sequential condensation/amine addition of the guests to bent-shape 2,3-dihyroquinazolinones, with a maximum observed rate acceleration of 38,000-fold. Because of their unfavorable nonplanar configurations, the products can be easily expelled from the cage cavities to allow multiple catalytic turnovers. The host-guest investigations between the cages and reactants/products have been examined by a variety of spectroscopic techniques and molecular dynamics simulations. Manipulation of steric and electronic properties of organic linkers in coordination cages can control guest uptake and release to realize high activities and selectivities, which will promote the design of new types of molecular containers for supramolecular catalysis.

\section{Methods}

Synthesis of the cages. A mixture of $\mathbf{L}_{1}$ or $\mathbf{L}_{2}(0.24 \mathrm{mmol})$, 2-formylpyridine (102 $\mathrm{mg}, 0.96 \mathrm{mmol})$, and $\mathrm{Zn}(\mathrm{OTf})_{2}(116 \mathrm{mg}, 0.32 \mathrm{mmol})$ was dissolved in $30 \mathrm{~mL}$ $\mathrm{CH}_{2} \mathrm{Cl}_{2}$ and $30 \mathrm{~mL} \mathrm{CH} \mathrm{CH}_{3} \mathrm{Cnd}$ then was heated at $70^{\circ} \mathrm{C}$ for $8 \mathrm{~h}$ to give a clear solution. Single crystals suitable for X-ray diffraction analysis were obtained by slow diffusion of a mixture of $\mathrm{Et}_{2} \mathrm{O}$ and THF or 1,4-dioxane and THF (2:1 v/v) into the solution at r.t. for 7 days. The product can be best formulated as $\left[\mathrm{Zn}_{8}\left(\mathbf{L}_{1}\right)_{6}\right]$ 16OTf.2THF. $3 \mathrm{CH}_{3} \mathrm{CN}$ for TPE-1 and $\left[\mathrm{Zn}_{8}\left(\mathrm{~L}_{2}\right)_{6}\right]$ 16OTf-4THF-8dioxane for TPE-2 on the basis of microanalysis, TGA, Q-TOF-MS, and IR.

TPE-1. Yield: $200 \mathrm{mg}$, about $64 \%$. Anal (\%). Calcd for $\mathrm{C}_{336} \mathrm{H}_{252} \mathrm{~N}_{52} \mathrm{Zn}_{8} \mathrm{~S}_{16} \mathrm{O}_{51} \mathrm{~F}_{48}$ : C, 59.16; H, 3.72; N, 10.68. Found: C, 59.23; H, 3.81; N, 10.86. ${ }^{1} \mathrm{H}$ NMR $\left(400 \mathrm{MHz}\right.$, DMSO- $\left.d_{6}\right) \delta: 8.73$ (bs, $\left.24 \mathrm{H}\right), 8.65$ (bs, 24H), $8.12(\mathrm{~s}$, $24 \mathrm{H}), 8.11(\mathrm{~s}, 24 \mathrm{H}), 7.62$ (bs, $24 \mathrm{H}), 7.20$ (bs, $48 \mathrm{H}), 7.07$ (bs, $48 \mathrm{H}) .{ }^{13} \mathrm{C}$ NMR $(100$ MHz, DMSO- $\left.d_{6}\right) \delta: 160.6,150.1,132.3,125.9,122.9,121.8,119.7,116.4,113.8$. IR $\left(\mathrm{KBr}\right.$ pellet, $\left.\mathrm{v} / \mathrm{cm}^{-1}\right): 1598(\mathrm{~m}), 1513(\mathrm{~m}), 1445(\mathrm{w}), 1267(\mathrm{vs}), 1162(\mathrm{~s}), 1031(\mathrm{~s})$, $914(\mathrm{w}), 839$ (w), 777 (w), 639 (m), 574 (s), 518 (m). Q-TOF-MS: m/z: 797.84 $\left[\mathrm{Zn}_{8}\left(\mathrm{~L}_{1}\right)_{6} \cdot 8 \mathrm{OTf} \cdot \mathrm{THF} \cdot 2 \mathrm{CH}_{3} \mathrm{CN}\right]^{8+}, 829.24\left[\mathrm{Zn}_{8}\left(\mathbf{L}_{1}\right)_{6} \cdot 8 \mathrm{OTf} \cdot 2 \mathrm{THF} \cdot 6 \mathrm{CH}_{3} \mathrm{CN} \cdot \mathrm{H}_{2} \mathrm{O}\right]^{8+}$, $963.19\left[\mathrm{Zn}_{8}\left(\mathbf{L}_{1}\right)_{6} \cdot 9 \mathrm{OTf} \cdot 4 \mathrm{THF} \cdot \mathrm{CH}_{3} \mathrm{CN} \cdot 2 \mathrm{H}_{2} \mathrm{O}\right]^{7+}, 1083.96\left[\mathrm{Zn}_{8}\left(\mathbf{L}_{1}\right)_{6} \cdot 10 \mathrm{OTf}\right]^{6+}$, $1331.55\left[\mathrm{Zn}_{8}\left(\mathbf{L}_{1}\right)_{6} \cdot 11 \mathrm{OTf}\right]^{5+}, 1700.41\left[\mathrm{Zn}_{8}\left(\mathbf{L}_{1}\right)_{6} \cdot 12 \mathrm{OTf}\right]^{4+}$.

TPE-2. Yield: $140 \mathrm{mg}$, about $35 \%$. Anal (\%). Calcd for

$\mathrm{C}_{508} \mathrm{H}_{471} \mathrm{~N}_{48} \mathrm{Zn}_{8} \mathrm{~S}_{16} \mathrm{O}_{62} \mathrm{~F}_{48}$ : C, 59.95; H, 4.61; N, 6.61. Found: C, 59.89; H, 4.75; N, 6.76. ${ }^{1} \mathrm{H}$ NMR $\left(400 \mathrm{MHz}\right.$, DMSO- $\left.d_{6}\right) \delta: 8.74(\mathrm{bs}, 48 \mathrm{H}), 8.15(\mathrm{bs}, 48 \mathrm{H}), 7.77(\mathrm{bs}$, $24 \mathrm{H}), 7.68-7.19(\mathrm{~m}, 192 \mathrm{H}) .{ }^{13} \mathrm{C}$ NMR $\left(100 \mathrm{MHz}\right.$, DMSO- $\left.d_{6}\right) \delta: 160.8,150.0,148.9$, $141.1,139.0,132.1,131.8,127.6,127.4,126.4,125,123.0,119.6,114.7$. IR ( $\mathrm{KBr}$ pellet, v/cm $\left.\mathrm{cm}^{-1}\right): 1599(\mathrm{~m}), 1494(\mathrm{~s}), 1445(\mathrm{w}), 1267(\mathrm{vs}), 1164(\mathrm{~m}), 1031(\mathrm{~s}), 1004$ (w), $823(\mathrm{~m}), 803(\mathrm{~m}), 776(\mathrm{w}), 639(\mathrm{~m}), 574(\mathrm{~s}), 517(\mathrm{w})$. Q-TOF-MS: m/z: 559.11 $\left[\mathrm{Zn}_{8}\left(\mathbf{L}_{2}\right)_{6} \cdot 3 \mathrm{OTf}\right]^{13+}, 650.41\left[\mathrm{Zn}_{8}\left(\mathbf{L}_{2}\right)_{6} \cdot 4 \mathrm{OTf} \cdot 4\left(1,4 \text {-dioxane) } \cdot \text { THF.CH } \mathrm{CH}_{3} \mathrm{CN}\right]^{12+}\right.$, 741.06 $\left[\mathrm{Zn}_{8}\left(\mathbf{L}_{2}\right)_{6} \cdot 5 \mathrm{OTf} \cdot 5 \text { (1,4-dioxane).THF.CH }{ }_{3} \mathrm{CN}\right]^{11+}, 795.89\left[\mathrm{Zn}_{8}\left(\mathbf{L}_{2}\right)_{6} \cdot 6 \mathrm{OTf} \cdot 2\right.$ (1,4-dioxane) $\left.\cdot \mathrm{CH}_{3} \mathrm{CN}\right]^{10+}, 916.64\left[\mathrm{Zn}_{8}\left(\mathbf{L}_{2}\right)_{6} \cdot 7 \mathrm{OTf} \cdot 3\left(1,4 \text {-dioxane) } \cdot \mathrm{THF} \cdot \mathrm{CH}_{3} \mathrm{CN}\right]^{9+}\right.$, $1267.19\left[\mathrm{Zn}_{8}\left(\mathbf{L}_{2}\right)_{6} \cdot 9 \mathrm{OTf} \cdot 7 \text { (1,4-dioxane).THF }\right]^{9+}$.

General procedure for the cage-based catalysis. To a solvent mixture of $\mathrm{CH}_{3} \mathrm{CN}$ and toluene $(1: 2 \mathrm{v} / \mathrm{v}, 3 \mathrm{~mL})$, anthranilamide $(0.68 \mathrm{mg}, 0.05 \mathrm{mmol})$, aldehyde $(0.055$ mmol), and TPE-1 $\left(0.4 \mathrm{mg}, 5 \times 10^{-5} \mathrm{mmol}\right)$ or TPE-2 $\left(0.5 \mathrm{mg}, 5 \times 10^{-5} \mathrm{mmol}\right)$ were added. The resultant solution was stirred at $40^{\circ} \mathrm{C}$ for $14 \mathrm{~h}$. The reaction mixture was cooled down to r.t. and extracted with ethyl acetate. The ethyl acetate solution was washed with water and dried over anhydrous sodium sulfate. The solvent was removed under reduced pressure and the crude products were separated by the column on silica gel (EtOAc/petroleum ether) to get the isolated yield.

General procedure for the multiple turnover catalysis. To a solution of the TPE$1\left(0.4 \mathrm{mg}, 5 \times 10^{-5} \mathrm{mmol}\right)$ or TPE-2 $\left(0.5 \mathrm{mg}, 5 \times 10^{-5} \mathrm{mmol}\right)$ in the mixed solvents of $\mathrm{CH}_{3} \mathrm{CN}(1 \mathrm{~mL})$ and toluene $(2 \mathrm{~mL})$, we added ten times successive portions of $3 \mathbf{a}$ $(0.05 \mathrm{mmol})$ and $\mathbf{4 b}(0.055 \mathrm{mmol})$, waiting until each aliquot had completely reacted before adding the next.

General procedure for $\mathbf{U V}$-Vis titration. The titration experiments were carried out by adding $30.0 \mu \mathrm{L}$ solution of substrates $\left(1.0 \times 10^{-3} \mathrm{~mol} \mathrm{~L}^{-1}\right)$ to a solution of TPE-1 or TPE-2 $\left(1.0 \times 10^{-5} \mathrm{~mol} \mathrm{~L}^{-1}\right)$ in $3.0 \mathrm{~mL} \mathrm{CH}_{3} \mathrm{CN}$ every $5 \mathrm{~min}$. The absorption was measured at room temperature.

Single-crystal X-ray crystallography. Single-crystal XRD data for TPE-1 and TPE-2 were collected on a Bruker D8 VENTURE CMOS photon 100 diffractometer with helios $\mathrm{mx}$ multilayer monochromator $\mathrm{Cu}$ Ka radiation $(\lambda=$ $1.54178 \AA$ ) at $173 \mathrm{~K}$. The empirical absorption correction was applied by using the SADABS program (G.M. Sheldrick, SADABS, program for empirical absorption correction of area detector data; University of Göttingen, Göttingen, Germany, 1996). The structure was solved by direct methods with SHELXS-2014 and refined with SHELXL-2014 using OLEX2-1.2. In both cages, all non-H atoms were subjected to anisotropic refinement by full matrix program. Contributions to scattering due to these highly disordered solvent molecules were removed using the SQUEEZE routine of PLATON. Structures were then refined again using the data generated. Crystal data and details of the data collection are given in Supplementary Table 1, and selected bond distances and angles are presented in Supplementary Tables $2-4$.

Molecular simulations. Molecular simulations were conducted to estimate the interaction energies for $\mathbf{3 a}$ and $\mathbf{4}(\mathbf{4 b}, \mathbf{4 e}$ or $\mathbf{4 f})$ in TPE-1 and TPE-2, respectively. The cages and reactants were described by the Lennard-Jones (LJ) and electrostatic potentials. The LJ potential parameters as listed in Supplementary Table 6 were adopted from the universal force field ${ }^{44}$, and the atomic charges of the cages and reactants were estimated using the Qeq method ${ }^{45}$. To calculate the interaction energy for $\mathbf{3} \mathbf{a}+\mathbf{4}$ in each cage, first, $\mathbf{3} \mathbf{a}$ and $\mathbf{4}$ together were inserted into TPE-1 or TPE-2, and followed by optimization using Materials Studio (Accelrys Inc., 2008); then, MD simulation was performed at $313 \mathrm{~K}$ for $15 \mathrm{~ns}$. The cage structure was assumed to be rigid during MD simulation, but all the reactant molecules were flexible and free to move. The LJ interactions were evaluated with a cutoff of $12 \AA$, and the electrostatic interactions were estimated using the Ewald summation method with an accuracy of $10^{-3} \mathrm{kcal} \mathrm{mol}^{-1}$

\section{Data availability}

The X-ray crystallographic coordinates for the structures reported in this article have been deposited at the Cambridge Crystallographic Data Centre (CCDC), under deposition numbers CCDC 1858566, 1858567, and 1858587. These data can be obtained free of charge from The Cambridge Crystallographic Data Centre via www.ccdc.cam.ac.uk/data_request/cif. All other data supporting the findings of this study are available within the article and its Supplementary Information, or from the corresponding author upon reasonable request.

Received: 19 April 2018 Accepted: 27 September 2018

Published online: 24 October 2018

\section{References}

1. Mal, P., Breiner, B., Rissanen, K. \& Nitschke, J. R. White phosphorus is airstable within a self-assembled tetrahedral capsule. Science 324, 1697-1699 (2009).

2. Xuan, W., Zhang, M., Liu, Y., Chen, Z. \& Cui, Y. A chiral quadruple-stranded helicate cage for enantioselective recognition and separation. J. Am. Chem. Soc. 134, 6904-6907 (2012).

3. Brown, C. J., Toste, F. D., Bergman, R. G. \& Raymond, K. N. Supramolecular catalysis in metal-ligand cluster hosts. Chem. Rev. 115, 3012-3035 (2015).

4. Samanta, S. K., Moncelet, D., Briken, V. \& Isaacs, L. Metal-organic polyhedron capped with Cucurbit [8] uril delivers doxorubicin to cancer cells. J. Am. Chem. Soc. 138, 14488-14496 (2016).

5. Zhang, Y. Y., Gao, W. X., Lin, L. \& Jin, G. X. Recent advances in the construction and applications of heterometallic macrocycles and cages. Coord. Chem. Rev. 344, 323-344 (2017). 
6. Cook, T. R. \& Stang, P. J. Recent developments in the preparation and chemistry of metallacycles and metallacages via coordination. Chem. Rev. 115, 7001-7045 (2015).

7. Yoshizawa, M., Tamura, M. \& Fujita, M. Diels-Alder in aqueous molecular hosts: unusual regioselectivity and efficient catalysis. Science 312, 251-254 (2006).

8. Nishioka, Y., Yamaguchi, T., Yoshizawa, M. \& Fujita, M. Unusual [2+4] and $[2+2]$ cycloadditions of arenes in the confined cavity of self-assembled cages. J. Am. Chem. Soc. 129, 7000-7001 (2007).

9. Pluth, M. D., Bergman, R. G. \& Raymond, K. N. Acid catalysis in basic solution: a supramolecular host promotes orthoformate hydrolysis. Science 316, 85-88 (2007).

10. Kaphan, D. M., Levin, M. D., Bergman, R. G., Raymond, K. N. \& Toste, F. D. A supramolecular microenvironment strategy for transition metal catalysis. Science 350, 1235-1238 (2015).

11. Inokuma, Y., Yoshioka, S. \& Fujita, M. A molecular capsule network: guest encapsulation and control of Diels-Alder reactivity. Angew. Chem. Int. Ed. 49, 8912-8914 (2010).

12. Ikemoto, K., Inokuma, Y. \& Fujita, M. Diels-Alder via molecular recognition in a crystalline molecular flask. J. Am. Chem. Soc. 133, 16806-16808 (2011).

13. Yoshizawa, M., Miyagi, S., Kawano, M., Ishiguro, K. \& Fujita, M. Alkane oxidation via photochemical excitation of a self-assembled molecular cage. $J$. Am. Chem. Soc. 126, 9172-9173 (2004).

14. Brown, C. J., Bergman, R. G. \& Raymond, K. N. Enantioselective catalysis of the aza-cope rearrangement by a chiral supramolecular assembly. J. Am. Chem. Soc. 131, 17530-17531 (2009).

15. Murase, T., Nishijima, Y. \& Fujita, M. Cage-catalyzed Knoevenagel condensation under neutral conditions in water. J. Am. Chem. Soc. 134, 162-164 (2012).

16. Hastings, C. J., Pluth, M. D., Bergman, R. G. \& Raymond, K. N. Enzymelike catalysis of the Nazarov cyclization by supramolecular encapsulation. J. Am. Chem. Soc. 132, 6938-6940 (2010).

17. Kaphan, D. M., Toste, F. D., Bergman, R. G. \& Raymond, K. N. Enabling new modes of reactivity via constrictive binding in a supramolecular-assemblycatalyzed Aza-Prins cyclization. J. Am. Chem. Soc. 137, 9202-9205 (2015).

18. García-Simón, C. et al. Enantioselective hydroformylation by a Rh-catalyst entrapped in a supramolecular metallocage. J. Am. Chem. Soc. 137, 2680-2687 (2015).

19. Lu, Y., Lin, Y. J., Li, Z. H. \& Jin., G. X. Highly stable molecular Borromean rings. Chin. J. Chem. 36, 106-111 (2018).

20. Jing, X., He, C., Yang, Y. \& Duan, C. A metal-organic tetrahedron as a redox vehicle to encapsulate organic dyes for photocatalytic proton reduction. J. Am. Chem. Soc. 137, 3967-3974 (2015).

21. Cullen, W., Misuraca, M. C., Hunter, C. A., Williams, N. H. \& Ward, M. D. Highly efficient catalysis of the Kemp elimination in the cavity of a cubic coordination cage. Nat. Chem. 8, 231-236 (2016).

22. Huang, S. L., Lin, Y. J., Andy Hor, T. S. \& Jin, G. X. Cp*Rh-based heterometallic metallarectangles: size-dependent Borromean link structures and catalytic acyl transfer. J. Am. Chem. Soc. 135, 8125-8128 (2013).

23. Li, H., Han, Y. F., Lin, Y. J., Guo, Z. W. \& Jin., G. X. Stepwise construction of discrete heterometallic coordination cages based on self-sorting strategy. J. Am. Chem. Soc. 136, 2982-2985 (2014).

24. Guo, J. et al. Regio- and enantioselective photodimerization within the confined space of a homochiral ruthenium/palladium heterometallic coordination cage. Angew. Chem. Int. Ed. 56, 3852-3856 (2017).

25. Centelles, M. V., Lawrence, L. A. \& Lusby, P. J. High activity and efficient turnover by a simple, self-assembled "artificial Diels-Alderase". J. Am. Chem. Soc. 140, 2862-2868 (2018).

26. Wasilke, J. C., Obrey, S. J., Baker, R. T. \& Bazan, G. C. Concurrent tandem catalysis. Chem. Rev. 105, 1001-1020 (2005).

27. Hayashi, Y. Pot economy and one-pot synthesis. Chem. Sci. 7, 866-880 (2016).

28. Ueda, Y., Ito, H., Fujita, D. \& Fujita, M. Permeable self-assembled molecular containers for catalyst isolation enabling two-step cascade reactions. J. Am. Chem. Soc. 139, 6090-6093 (2017).

29. Jiao, J. J. et al. Design and assembly of chiral coordination cages for asymmetric sequential reactions. J. Am. Chem. Soc. 140, 2251-2259 (2018)

30. Kennedy, C. R., Lin, S. \& Jacobsen, E. N. The cation $-\pi$ interaction in smallmolecule catalysis. Angew. Chem., Int. Ed. 55, 12596-12624 (2016).

31. Zhao, Z., Lam, J. W. Y. \& Tang, B. Z. Tetraphenylethene: a versatile AIE building block for the construction of efficient luminescent materials for organic light-emitting diodes. J. Mater. Chem. 22, 23726-23740 (2012).

32. Yan, X. et al. Light-emitting superstructures with anion effect: coordinationdriven self-assembly of pure tetraphenylethylene metallacycles and metallacages. J. Am. Chem. Soc. 138, 4580-4588 (2016).

33. Zhou, Z. et al. Immobilizing tetraphenylethylene into fused metallacycles: shape effects on fluorescence emission. J. Am. Chem. Soc. 138, 13131-13134 (2016).

34. Lu, J. \& Zhang, J. Facile synthesis of azo-linked porous organic frameworks via reductive homocoupling for selective $\mathrm{CO}_{2}$ capture. J. Mater. Chem. A 2, 13831-13834 (2014)
35. Huang, D., Li, X., Xu, F., Li, L. \& Lin, X. Highly enantioselective synthesis of dihydroquinazolinones catalyzed by SPINOL-phosphoric acids. ACS Catal. 3 , 2244-2247 (2013).

36. Pluth, M. D., Bergman, R. G. \& Raymond, K. N. Supramolecular catalysis of orthoformate hydrolysis in basic solution: an enzyme-like mechanism. J. Am. Chem. Soc. 130, 11423-11429 (2008).

37. Prakash, M., Jayakumar, S. \& Kesavan, V. Investigation of the enantioselective synthesis of 2,3-dihydroquinazolinones using Sc(III)-inda-pybox. Synthesis 45, 2265-2272 (2013)

38. Chen, J. X. et al. Gallium(III) triflate-catalyzed one-pot selective synthesis of 2,3-dihydroquinazolin-4(1H)-ones and quinazolin-4(3H)-ones. Tetrahedron Lett. 49, 3814-3818 (2008).

39. Salehi, P., Dabiri, M., Zolfigol, M. A. \& Baghbanzadeh, M. A novel method for the one-pot three-component synthesis of 2,3-dihydroquinazolin-4(1H)-ones. Synlett 7, 1155-1157 (2005).

40. Mugridge, J. S., Szigethy, G., Bergman, R. G. \& Raymond, K. N. Encapsulated guest-host dynamics: guest rotational barriers and tumbling as a probe of host interior cavity space. J. Am. Chem. Soc. 132, 16256-16264 (2010)

41. Akine, S., Miyashita, M. \& Nabeshima, T. A metallo-molecular cage that can close the apertures with coordination bonds. J. Am. Chem. Soc. 139, 4631-4634 (2017).

42. Mugridge, J. S., Bergman, R. G. \& Raymond, K. N. 1 H NMR chemical shift calculations as a probe of supramolecular host-guest geometry. J. Am. Chem. Soc. 133, 11205-11212 (2011)

43. Yin, Q., Klare, H. \& Oestreich, M. Catalytic Friedel-Crafts $\mathrm{C}-\mathrm{H}$ borylation of electron-rich arenes: dramatic rate acceleration by added alkenes. Angew. Chem. Int. Ed. 56, 3712-3717 (2017).

44. Rappe, A. K. \& Goddard, W. A. Charge equilibration for molecular dynamics simulations. J. Phys. Chem. 95, 3358-3363 (1991).

45. Rappé, A. K. et al. A full periodic table force field for molecular mechanics and molecular dynamics simulations. J. Am. Chem. Soc. 114, 10024-10035 (1992).

\section{Acknowledgements}

This work was financially supported by the National Science Foundation of China (Grants 21522104, 21431004, and 21620102001), the National Key Basic Research Program of China (Grants 2014CB932102 and 2016YFA 0203400), Key Project of Basic Research of Shanghai (17JC1403100 and 18JC1413200), and the Shanghai "Eastern Scholar" Program.

\section{Author contributions}

Y.C. and Y.L. conceived and designed the research. J.J.J. and J.Q.D. performed the experiments. Z.J.L. solved the crystal structures. J.W.J., Z.W.Q. and X.L. conducted theoretical calculations. Y.C., Y.L. and J.J.J. analyzed the data and co-wrote the manuscript.

\section{Additional information}

Supplementary Information accompanies this paper at https://doi.org/10.1038/s41467018-06872-0.

Competing interests: The authors declare no competing interests.

Reprints and permission information is available online at http://npg.nature.com/ reprintsandpermissions/

Publisher's note: Springer Nature remains neutral with regard to jurisdictional claims in published maps and institutional affiliations.

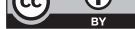

Open Access This article is licensed under a Creative Commons Attribution 4.0 International License, which permits use, sharing, adaptation, distribution and reproduction in any medium or format, as long as you give appropriate credit to the original author(s) and the source, provide a link to the Creative Commons license, and indicate if changes were made. The images or other third party material in this article are included in the article's Creative Commons license, unless indicated otherwise in a credit line to the material. If material is not included in the article's Creative Commons license and your intended use is not permitted by statutory regulation or exceeds the permitted use, you will need to obtain permission directly from the copyright holder. To view a copy of this license, visit http://creativecommons.org/ licenses/by/4.0/.

(c) The Author(s) 2018 